\title{
Adherence to artemether/lumefantrine treatment in children under real-life situations in rural Tanzania
}

\author{
D.O. Simba \\ Muhimbili University of Health and Allied Sciences \\ D. Kakoko \\ Muhimbili University of Health and Allied Sciences \\ G. Tomson \\ Karolinska Institutet \\ Zul Premji \\ Aga Khan University, zul.premji@aku.edu \\ M. Petzold \\ Karolinska Institutet
}

See next page for additional authors

Follow this and additional works at: https://ecommons.aku.edu/eastafrica_fhs_mc_pathol

Part of the Pathology Commons

\section{Recommended Citation}

Simba, D., Kakoko, D., Tomson, G., Premji, Z., Petzold, M., Mahindi, M., Gustafsson, L. (2012). Adherence to artemether/lumefantrine treatment in children under real-life situations in rural Tanzania. Transactions of the Royal Society of Tropical Medicine and Hygiene, 106(1), 3-9.

Available at: https://ecommons.aku.edu/eastafrica_fhs_mc_pathol/50 


\section{Authors}

D.O. Simba, D. Kakoko, G. Tomson, Zul Premji, M. Petzold, M. Mahindi, and L.L. Gustafsson 


\title{
Adherence to artemether/lumefantrine treatment in children under real-life situations in rural Tanzania
}

\author{
Daudi O. Simba ${ }^{\mathrm{a}, \mathrm{b}, *}$, Deodatus Kakoko ${ }^{\mathrm{c}}$, Goran Tomson ${ }^{\mathrm{b}, \mathrm{d}}$, Zul Premji ${ }^{\mathrm{e}}$, Max Petzold ${ }^{\mathrm{b}, \mathrm{f}}$, \\ Margarita Mahindi ${ }^{g}$, Lars L. Gustafsson ${ }^{\mathrm{g}}$ \\ a Department of Community Health, Muhimbili University of Health and Allied Sciences, Dar es Salaam, Tanzania \\ b Division of Global Health (IHCAR), Department of Public Health Sciences, Karolinska Institutet, Stockholm, Sweden \\ c Department of Behavioural Sciences, Muhimbili University of Health and Allied Sciences, Dar es Salaam, Tanzania \\ d Medical Management Centre, Karolinska Institutet, Stockholm, Sweden \\ e Department of Parasitology and Medical Entomology, Muhimbili University of Health and Allied Sciences, Dar es Salaam, Tanzania \\ f Nordic School of Public Health, Göteborg, Sweden \\ g Unit of Tropical Pharmacology, Division of Clinical Pharmacology, Department of Laboratory Medicine, Karolinska University Hospital, Huddinge, \\ Stockholm, Sweden
}

\section{A R T I C L E I N F O}

\section{Article history:}

Received 15 November 2010

Received in revised form

12 September 2011

Accepted 12 September 2011

Available online 17 November 2011

\section{Keywords:}

Adherence

Artemisinin-based combination therapy

Health systems

Blood lumefantrine analysis

Under-five children

Rural community

\begin{abstract}
A B S T R A C T
A follow-up study was conducted to determine the magnitude of and factors related to adherence to artemether/lumefantrine (ALu) treatment in rural settings in Tanzania. Children in five villages of Kilosa District treated at health facilities were followed-up at their homes on Day 7 after the first dose of ALu. For those found to be positive using a rapid diagnostic test for malaria and treated with ALu, their caretakers were interviewed on drug administration habits. In addition, capillary blood samples were collected on Day 7 to determine lumefantrine concentrations. The majority of children $(392 / 444 ; 88.3 \%)$ were reported to have received all doses, in time. Non-adherence was due to untimeliness rather than missing doses and was highest for the last two doses. No significant difference was found between blood lumefantrine concentrations among adherent (median $286 \mathrm{nmol} / \mathrm{l}$ ) and nonadherent [median $261 \mathrm{nmol} / \mathrm{l}$; range $25 \mathrm{nmol} / 1$ (limit of quantification) to $9318 \mathrm{nmol} / \mathrm{l}$ ]. Children from less poor households were more likely to adhere to therapy than the poor [odds ratio $(\mathrm{OR})=2.45,95 \% \mathrm{CI} 1.35-4.45$; adjusted $\mathrm{OR}=2.23,95 \% \mathrm{CI} 1.20-4.13$ ]. The high reported rate of adherence to ALu in rural areas is encouraging and needs to be preserved to reduce the risk of emergence of resistant strains. The age-based dosage schedule and lack of adherence to ALu treatment guidelines by health facility staff may explain both the huge variability in observed lumefantrine concentrations and the lack of difference in concentrations between the two groups.
\end{abstract}

(C) 2011 Royal Society of Tropical Medicine and Hygiene. Published by Elsevier Ltd. All rights reserved.

\section{Introduction}

In 2006, Tanzania switched from sulfadoxine/ pyrimethamine (SP) to artemether/lumefantrine (ALu) as

\footnotetext{
* Corresponding author. Present address: P.O. Box 65015, Dar es Salaam, Tanzania. Tel.: +25522 215 0304/5; fax: +25522 2153114 .

E-mail addresses: dsimba@muhas.ac.tz,daudisimba@yahoo.com (D.O. Simba)
}

the first-line treatment recommended for uncomplicated falciparum malaria. The limited availability of ALu, combined with its rapid onset of action and rapid clearance of severe clinical symptoms, might tempt caretakers to discontinue treatment prematurely ${ }^{1}$ and to keep the remaining medicine for an 'anticipated' next attack. ${ }^{2}$ This may accelerate the pace of development of drug resistance, ${ }^{3}$ thus making it important to maintain high adherence rates to preserve the efficacy of 
artemisinin-based combination therapy (ACT) in Africa and elsewhere. ${ }^{4}$

Adherence levels as high as $90 \%$ have been reported in controlled clinical trials. ${ }^{5,6}$ These figures are likely to be an overestimation because in real-life situations caretakers are neither given detailed instruction on to how to administer the treatment nor do they receive follow-up on the use of the drugs by the child.

Two community-based Tanzanian studies conducted in Kilombero/Ulanga Districts and Zanzibar Islands did report high levels of adherence to ACT treatment regimens (77\% and $99 \%$, respectively). ${ }^{7,8}$ However, more information on adherence to ALu is required in order to inform the implementation of drug policy change, given the low access to ALu in rural remote areas. ${ }^{9}$ Community-based data collected under real-life settings are also required in order to estimate the level of community effectiveness, ${ }^{10,11}$ which reflects the success of implementation of a drug policy change.

Several factors have been reported to influence adherence to treatment, including: education of caretakers and the use of pre-packaged co-blister packets ${ }^{12,13}$; drug efficacy and rapid improvement of symptoms ${ }^{1,3,14}$; local perceptions about the disease ${ }^{15}$; and caretakers perceiving a child playing after a single dose as a sign of cure. ${ }^{16}$ Most of these findings, however, are based on studies performed in Asia or during the time when chloroquine (CQ) and SP were the first-line recommended drugs. ${ }^{7,8}$ This community-based study was therefore conducted to determine the level of and factors influencing adherence to ALu among under-fives in a real-life situation in rural Tanzania. Information from this study will be important in designing appropriate implementation programmes for ACT in Tanzania and elsewhere in Africa. ${ }^{5}$

\section{Materials and methods}

\subsection{Study site}

A 12-month follow-up study was conducted in June 2008 in Kilosa District, Tanzania. A detailed description of the district is reported elsewhere. ${ }^{9}$ Malaria accounts for more than one-half of clinical presentations by patients attending the outpatient clinics and $60 \%$ of deaths among under-five hospital admissions (district annual report, 2008). The health facilities are organised at three levels, with 61 dispensaries that are at the lowest contact level, seven health centres at the middle, and two hospitals as well as a district hospital at the highest level. Only hospitals and health centres have a functioning microscope for malaria diagnosis. Dispensaries have a catchment population of approximately 10000 people, manned by a clinician and a public health nurse, and serve the majority of the children suspected to have uncomplicated malaria.

\subsection{Study design and sample size}

This was a follow-up observational study. Stratified cluster sampling was carried out. All 30 rural villages listed as rural in the National Census Report, 2003, located in the wet-lowland areas, were grouped into those with and without a health facility. One village among 12 villages with a health facility and two villages among 18 villages without a health facility were randomly selected using EPI Info v.6 (CDC, Atlanta, GA, USA). The rationale of $1: 2$ was based on the proportion of villages with health facilities in the district. During the course of data collection, it was necessary to compare between rural and semi-urban villages. Thus, the ward headquarters of the three rural villages located in semi-urban areas were included in the study. Two of the initial villages shared the same ward; therefore, two semi-urban villages were added making the total number of study villages equal to five (Figure 1).

The unadjusted sample size was calculated as 280 based on the assumption that $24 \%$ of caretakers would adhere to treatment, as reported in a study in Uganda. ${ }^{11}$ Although this study was based on CQ treatment, this was the most recent adherence study in a setting similar to Tanzania. A maximum $95 \% \mathrm{CI}$ of $\pm 5 \%$ was required, and adding $10 \%$ for possible loss to follow-up and another $27 \%$ as a correction factor for clustering effect (intracluster correlation coefficient $=0.01$ ), the sample size was calculated as 391 , approximated to 400 . Based on Tanzanian studies, it was assumed that approximately $80-90 \% 17,18$ of children with fever would be treated at health centres or dispensaries. Data collection was planned to be completed in a 4 -month period. However, during the study it was found that most caretakers residing in villages without a health facility sought care from drug vendors and ordinary shops instead. ${ }^{9}$ Consequently, it took 12 months to recruit the required sample size.

\subsection{Data collection}

Three research assistants (RA) were purposely recruited both to administer the questionnaire and to take blood samples. They were taught for 6 days on how to administer the questionnaire and take blood samples.

At the time of the study, there were only three health facilities in the study area that provided ALu, all of which were public facilities. Therefore, these health facilities acted as enrolment centres where RAs identified the names of children who met the enrolment criteria from the laboratory and outpatient registers.

The enrolment criteria of children for the study were:

- child aged 3-59 months;

- child diagnosed at the health facility as positive for malaria using a rapid diagnostic test (RDT);

- child prescribed with the correct dose of ALu according to national treatment guideline, which is based on weight and age, twice daily for 3 days ${ }^{19}$; and

- child resided in one of the five selected villages.

RAs traced the children home on Day 7 after the first dose of ALu. Recruitment of children into the study was performed after a caretaker had provided written informed consent. Caretakers were not given prior information about the follow-up. Caretakers were interviewed regarding the instructions received at the facility, the day and time they administered the drugs, whether food was taken with the drugs, whether food was cooked with oil, and whether 


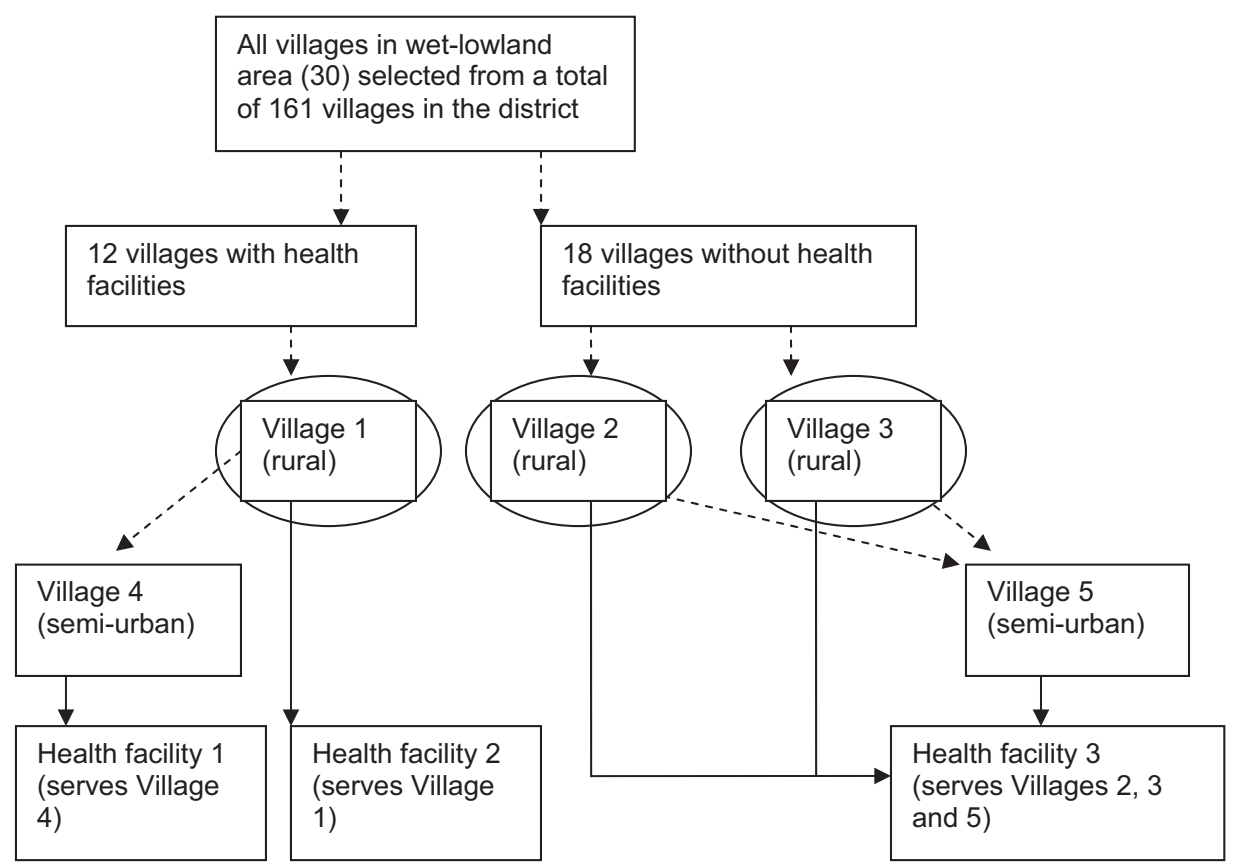

Figure 1. Overview of the process to recruit 5 out of 161 villages and study subjects $(n=446)$ in Kilosa District, Tanzania.

the child vomited, and if so, how soon after the drug intake. (The questionnaire can be obtained from the corresponding author upon request.) Two caretakers could not remember the events and were excluded from the analysis. In addition, baseline data on demographic and socioeconomic status (SES) and knowledge about malaria treatment were obtained. The questionnaire was based on a literature review of previous adherence studies. ${ }^{6,11}$ A pilot study was carried out for 1 week in two villages to validate the questionnaire and to gain field experiences in blood sampling techniques.

\subsubsection{Definition of adherence to}

\section{artemether/lumefantrine treatment schedule}

Adherence to treatment with ALu was defined as a caretaker reported to have administered two doses daily for 3 days; if the first dose was started in the evening, then one dose on the first day, two doses for 2 days and the last dose on the fourth day, in the morning. The dose was one tablet of ALu (20 mg artemether and $120 \mathrm{mg}$ lumefantrine) for a child aged $<3$ years and two tablets (artemether $40 \mathrm{mg}$ and lumefantrine $240 \mathrm{mg}$ ) for a child aged $\geq 3$ years.

\subsection{Blood sampling, handling and drug sample analysis}

RAs collected capillary blood samples from the children on Day 7 through a finger-prick using the method previously described by Ntale et al. ${ }^{20}$ After drying the filter paper, the specimen was stored in a plastic envelope to avoid contamination and was collected by the supervisors weekly. Filter paper samples were stored at room temperature for 3-15 months at the Pharmacology Laboratory of Muhimbili University (Dar es Salaam, Tanzania) before transfer to the Clinical Pharmacology Laboratory at Karolinska Institutet (Stockholm, Sweden) where blood lumefantrine concentrations were determined by HPLC as described by Ntale et al. ${ }^{20}$ Samples were assayed with an intra-assay and inter-assay coefficient of variation of $<9.2 \%$ and with a limit of quantification (LoQ) of $25 \mathrm{nmol} / \mathrm{l}$.

At the time of the study, several pilot studies were ongoing in the country aimed at rolling out of RDTs for malaria. We therefore introduced RDTs for malaria diagnosis in the three facilities, since Kilosa was not one of the pilot districts. Health workers in each facility and village health workers in two dispensaries that were understaffed were trained in performing the RDT for malaria. A 1-day training course was held followed by 2 days of work under supervision. The tests were performed routinely for all children under five with a suspected malaria diagnosis irrespective of whether they resided in the five study villages or not. The RDT called Paracheck(Orchid Biomedical Systems, Goa, India) was obtained through donation from the National Malaria Control Programme.

\subsection{Data analysis}

Data were double-entered into EpiData 3.0 (EpiData Association, Odense, Denmark) before being transferred to Stata v.10.1 (StataCorp., College station, TX, USA). Bivariate analysis was performed on variables thought to influence adherence to treatment with ALu. Variables found to be associated were introduced in a stepwise multiple regression model using a cut-off point of 0.10 . The variables were: knowledge of treatment of severe malaria; SES; and intake of food with oil. Marital status and age of the child were also included. The variables SES and intake of food with oil remained in the final regression model; these were tested for interaction and no association was found. SES was calculated using principal component analysis in which 
Table 1

Background demographic characteristics of children under five enrolled in the study and their caretakers by reported adherence status $(n=444)$

\begin{tabular}{|c|c|c|c|c|}
\hline Characteristic of parent or child & $\begin{array}{l}\text { Reported non-adherence } \\
n(\%)\end{array}$ & $\begin{array}{l}\text { Reported adherence } \\
n(\%)\end{array}$ & Total & $P$-value \\
\hline \multicolumn{5}{|l|}{ Caretaker's age (years) } \\
\hline$<30$ & $32(12.3)$ & $228(87.7)$ & 260 & \multirow[t]{2}{*}{0.642} \\
\hline$\geq 30$ & $20(10.9)$ & $164(89.1)$ & 184 & \\
\hline \multicolumn{5}{|l|}{ Child's age (years) } \\
\hline$<2$ & $26(14.1)$ & $159(85.9)$ & 185 & \multirow[t]{2}{*}{0.195} \\
\hline$\geq 2$ & $26(10.0)$ & $233(90.0)$ & 259 & \\
\hline \multicolumn{5}{|l|}{ Child's sex } \\
\hline Male & $30(13.7)$ & $189(86.3)$ & 219 & \multirow[t]{2}{*}{0.199} \\
\hline Female & $22(9.8)$ & $203(90.2)$ & 225 & \\
\hline \multicolumn{5}{|l|}{ Caretaker's marital status } \\
\hline Not married & $34(14.3)$ & $204(85.7)$ & 238 & \multirow[t]{2}{*}{0.070} \\
\hline Married & $18(8.7)$ & $188(91.3)$ & 206 & \\
\hline \multicolumn{5}{|l|}{ Caretaker's education (years) } \\
\hline$<7$ & $25(13.4)$ & $162(86.6)$ & 187 & \multirow[t]{2}{*}{0.354} \\
\hline$\geq 7$ & $27(10.5)$ & $230(89.5)$ & 257 & \\
\hline \multicolumn{5}{|l|}{ Residence } \\
\hline Rural & $40(12.5)$ & $281(87.5)$ & 321 & \multirow[t]{2}{*}{0.428} \\
\hline Semi-urban & $12(9.8)$ & $111(90.2)$ & 123 & \\
\hline \multicolumn{5}{|l|}{ Village status } \\
\hline Without a health facility & $22(13.0)$ & $147(87.0)$ & 169 & \multirow[t]{3}{*}{0.502} \\
\hline With a health facility & $30(10.9)$ & $245(89.1)$ & 275 & \\
\hline Overall & $52(11.7)$ & $392(88.3)$ & 444 & \\
\hline
\end{tabular}

19 assets adopted from the Tanzania Demographic Health Survey were used. ${ }^{21}$ Variables that had $\geq 90 \%$ of the study children in one category were excluded. The final list included household construction material composing walls and the roof; the source of drinking water and type of light; and ownership of a watch or clock, radio, mattress, a bednet and a bicycle. The first component explained $30 \%$ of the variability and was used to construct a SES index. The SES index was categorised in five quintiles, and households in the first and second quintiles were classified as poor and the others as less poor. Crude and adjusted odds ratios (OR) with $95 \%$ CIs were reported.

Blood lumefantrine concentrations were found to have a positive skewed distribution. Hence, log-transformed values of lumefantrine concentrations were used for statistical testing of difference in mean levels ( $t$-test) between children who adhered to therapy and those who did not. The original concentration levels were also described using medians and box plot. Before and after comparison of blood lumefantrine concentrations could not be done because this was an observational study that aimed at determining adherence to ALu under real-life conditions. Blood sampling for determination of lumefantrine concentrations was not done routinely at the health facilities. Permission to conduct the study was obtained from the regional and district authorities. Written informed consent was obtained prior to interviews from each adult caretaker during baseline data collection. All caretakers approached provided informed consent for the study, except 18 who refused to have a blood sample taken from their children.

\section{Results}

A total of 544 names of eligible children were identified at the health facilities, 467 of whom were found on Day 7 and their caretakers interviewed; 77 (14.2\%) of the children were not included because they were not found at home (70), they were $>5$ years of age (2), they did not receive ALu (4) or they had died (1). Blood samples for lumefantrine concentrations were collected on Day 7 from 446 children; blood samples could not be collected from 21 children because their caretakers refused (18), the RAs failed to draw a second sample after the first attempt failed (2) or the capillary tube broke (1). Two caretakers could not recall most of the information; therefore, analysis was carried out based on the 444 children who had complete data.

Table 1 demonstrates no difference in sociodemographic characteristics between children who adhered to ALu treatment and those who did not. More than one-half of the children $(58.3 \%)$ were aged $\geq 2$ years and over onehalf of the caretakers (57.9\%) had at least primary level education.

The level of reported adherence to ALu was high (392/444; 88.3\%). Figure 2 shows that the number of

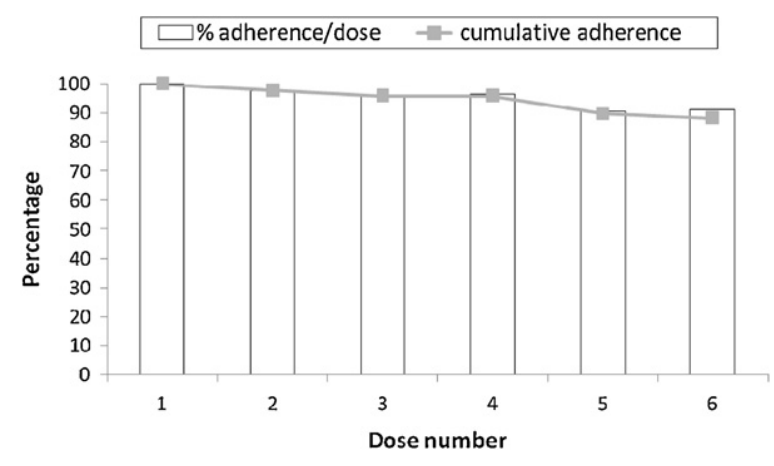

Figure 2. Reported percent adherence rate by caretakers for each of the six doses and cumulative adherence from the first to sixth dose $(n=444)$. 
Table 2

Logistic regression analysis of factors influencing adherence to artemether/lumefantrine (ALu) among children under five in Kilosa District, Tanzania $(n=444)$

\begin{tabular}{|c|c|c|c|c|}
\hline Factor & OR & $95 \% \mathrm{CI}$ & Adjusted OR & $95 \% \mathrm{CI}$ \\
\hline \multicolumn{5}{|l|}{ Access to healthcare services } \\
\hline Child's age $\geq 2$ years & 1.47 & $0.82-2.61$ & 1.80 & $0.90-3.58$ \\
\hline Married & 1.74 & $0.95-3.19$ & 1.87 & $0.90-3.88$ \\
\hline Caretaker's basic education $\geq 7$ years & 1.32 & $0.74-2.35$ & & \\
\hline Residing in semi-urban area & 1.32 & $0.67-2.60$ & & \\
\hline Using own or hired bicycle to go to health facility & 1.16 & $0.58-2.29$ & & \\
\hline Distance $<5 \mathrm{~km}$ from a government facility & 1.46 & $0.81-2.63$ & & \\
\hline \multicolumn{5}{|l|}{ Knowledge on malaria } \\
\hline Knowledge that mosquitoes cause malaria & 1.65 & $0.65-4.17$ & & \\
\hline Knowledge that high fever causes convulsion in children & 1.41 & $0.76-2.60$ & & \\
\hline Knowledge of treatment of severe malaria & 0.45 & $0.20-1.01$ & & \\
\hline $\begin{array}{l}\text { Knowledge that dawa mseto (ALu) is the recommended drug for the } \\
\text { treatment of malaria ya kawaida (uncomplicated malaria) }\end{array}$ & 0.73 & $0.39-1.37$ & & \\
\hline \multicolumn{5}{|l|}{ Socioeconomic status } \\
\hline Less poor (quintiles 3-5) & 2.45 & $1.35-4.45$ & 2.23 & $1.20-4.13$ \\
\hline \multicolumn{5}{|l|}{ Other factors } \\
\hline History of vomiting during malaria episode & 0.68 & $0.22-2.06$ & & \\
\hline Taken food with oil & 4.62 & $2.53-8.41$ & 4.60 & $2.49-8.50$ \\
\hline Taken adequate amount of food & 1.08 & $0.59-1.98$ & & \\
\hline Sought treatment same or next day & 0.97 & $0.51-1.85$ & & \\
\hline
\end{tabular}

OR: odds ratio.

children who were reported to have taken all doses, in time, was lowest for the last two doses.

When controlling for other factors, children from less poor households were more likely to report adherence to ALu treatment compared with the poor $(\mathrm{OR}=2.45,95 \% \mathrm{CI}$ 1.35-4.45; adjusted $\mathrm{OR}=2.23,95 \% \mathrm{CI}=1.20-4.13$ ) (Table 2 ). Children who took food with oil were also more likely to adhere to ALu treatment compared with those who did not $(\mathrm{OR}=4.62,95 \% \mathrm{CI} 2.53-8.41$; adjusted $\mathrm{OR}=4.60$, 95\% CI 2.49-8.50). Caretakers from the third SES quintile were most likely to adhere to treatment compared with the first quintile, however there was no observed trend.

Although the majority of caretakers (409/444; 92.1\%) reported being advised on the frequency and duration for ALu administration, the knowledge did not significantly influence the reported adherence. Basic education, offered for 7 years in the country, did not influence adherence to the treatment schedule.

No significant difference in mean blood lumefantrine concentrations was found between children whose caretakers reported adherence and those who reported non-adherence. The corresponding median levels were $286 \mathrm{nmol} / \mathrm{l}$ and $261 \mathrm{nmol} / \mathrm{l}$, respectively (Figure 3). However, there was a wide variation in blood lumefantrine concentrations, ranging from $25 \mathrm{nmol} / \mathrm{l}$ (LoQ) to $9318 \mathrm{nmol} / \mathrm{l}$ for the total group, and from $25 \mathrm{nmol} / 1$ to $1382 \mathrm{nmol} / \mathrm{l}$ for those with reported non-adherence and from $25 \mathrm{nmol} / \mathrm{l}$ to $9318 \mathrm{nmol} / 1$ for those with reported adherence. Extreme values (outliers) are not shown in Figure 3.

\section{Discussion}

This study has shown a high (88\%) level of reported adherence to ALu treatment under real-life settings, contrary to fears that efficacy observed in controlled trials might not be reflected in real-life situations. ${ }^{22}$ Similar findings were reported in studies performed in Kilombero/ Ulanga Districts and in Zanzibar Islands, Tanzania, despite some methodological differences. Whereas these studies included patients residing closer to health facilities (5-6 km from health facilities), mostly in urban and semiurban areas and applied presumptive malaria diagnosis, ${ }^{9}$ the current included children from rural remote areas (up to $25 \mathrm{~km}$ away) where the burden of malaria is the highest. $^{23}$

Therefore, the current study confirms a high adherence to ALu among under fives diagnosed to have malaria using a RDT even in most rural settings remote from health facilities.

No significant difference in blood lumefantrine concentrations was found between children reported to adhere to therapy and those who did not, in line with a study in Malawi ${ }^{24}$ but contrary to a study in Uganda. ${ }^{6}$ One of the reasons for the lack of any difference might be due to the lack of a clear cut-off point. ${ }^{24}$ Although a previous study in Thailand reported a Day 7 blood lumefantrine

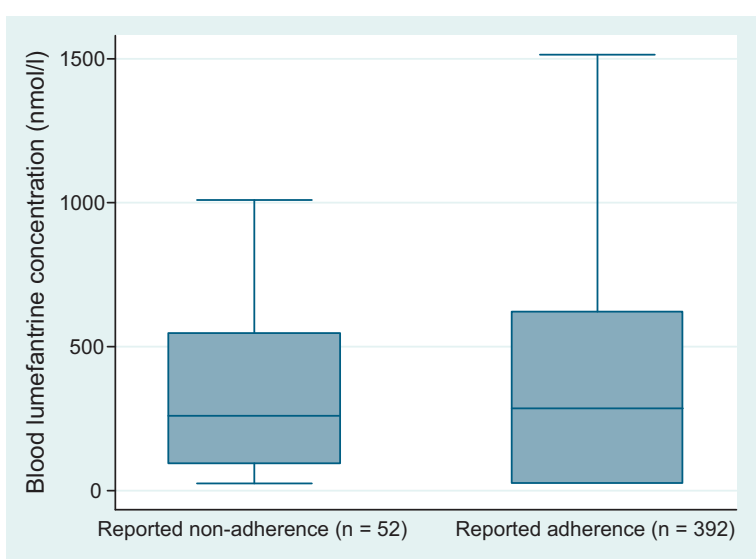

Figure 3. Distribution of lumefantrine blood concentration levels of study children by reported adherence status $(n=444)$. Outliers not shown in box plot. 
concentration cut-off point of $331 \mathrm{nmol} / \mathrm{l}(175 \mathrm{ng} / \mathrm{ml}),{ }^{25}$ another study in Malawi reported patients were cured even below this level. ${ }^{24}$ The difference might be explained by possible variations in food intake, ${ }^{26}$ as the levels of lumefantrine blood concentrations may increase 16 -fold in patients who took the drug with fatty food compared with without fat. 27,28 Age-based dosage schedules of ALu might also explain the unpredictable blood concentrations of lumefantrine, since the dose per kg may vary widely between patients, as reported for the $\mathrm{CQ} / \mathrm{SP}$ combination. ${ }^{29}$ This underscores the need to monitor the rate of adherence to treatment guidelines by providers as well as treatment schedules among patients in rural areas when implementing ALu treatment on a wide scale in Africa.

The high reported adherence found in this study can be explained by the availability of ALu in pre-packaged packets with pictorial instructions, which have been reported to improve caretakers' understanding of the schedule administration of the drug ${ }^{30}$ and hence they are more likely to follow the provider's advice. ${ }^{12,22,31}$ Studies conducted when CQ resistance was high reported low adherence levels, ${ }^{10,11}$ thus the highly efficacious ALu used in the present study might also explain the high reported level of adherence to treatment. ${ }^{22}$

Non-adherence to ALu treatment found in this study was mostly attributed to untimely dosing rather than missing doses, contrary to concerns that caretakers might discontinue treatment and save the remaining drugs for later use. ${ }^{1,2}$ The high level of non-adherence in the last two doses has also been reported in previous studies in Tanzania and Malawi. ${ }^{8,24}$ This might be due to some caretakers perceiving that a child playing after an initial dose is cured. ${ }^{16}$ Encouragingly, the low non-adherence level might have little impact on the treatment strategy; however, there is a potential risk of building up pressure for resistant strains over time. ${ }^{32}$

Children from the less poor households were found to adhere to treatment better than poor patients. This can partly be explained by the higher level of education among caretakers in the less poor households and the tendency to own radios compared with the poor. ${ }^{9}$ This might have exposed them to more information disseminated by the National Malaria Control Programme through radios and posters since the introduction of ALu.

\subsection{Methodological issues}

Caretakers' reports are subjective and were likely to overestimate the level of reported adherence owing to possibilities of obtaining socially desirable responses. ${ }^{24}$ This was minimised by explaining to the caretaker the objective of the study prior to the interviews. The fact that most children were no longer sick on Day 7 reduced the likelihood of receiving desirable responses. This is in contrast to reports of limited validity of caretakers' reports when interviewed at the health facilities. ${ }^{33}$ Pill count could not be performed on Day 7 since most of the caretakers had lost the packets. This limited the opportunity to triangulate study findings. However, the fact that the results conform to other community-based studies conducted in Tanzania where pill count was performed indicates that misreporting might be minimal.

Recalling detailed information about how the drug was administered after 7 days might result in data inaccuracy as some details might be forgotten. We tried to minimise this by following-up children weekly when the caretakers could still remember the details. In addition, a sick child tends to draw the attention of caretakers in a household, thus increasing the likelihood of remembering events in more details.

Blood lumefantrine concentrations would be useful when studying adherence. However, the lack of a clear cutoff point ${ }^{24}$ and baseline levels limited a detailed analysis. There were also other challenges resulting from applying the method in a real-life situation. Several methodological issues were found in this study that could limit the usefulness of the capillary blood sampling method for lumefantrine in evaluating ALu effectiveness under real-life conditions. These included the lack of baseline lumefantrine concentration, inability to control or record food intake, and inconsistency in determining ALu dosage where some prescribers based the dosage on child weight while others used age. In addition, some prescribers used child weight measured on the same day while others used the record found in the growth monitoring card, which could be outdated by several weeks or months.

\section{Conclusion}

This study showed that the reported rate of adherence to ALu treatment was encouragingly high in rural villages with or without a health facility. The large variability in lumefantrine concentrations cast doubt on the rationale of using aged-based dosage schedules of ALu as presently used in some facilities in Tanzania. Nonadherence was mainly attributed to off-schedule dosing rather than taking a fewer number of doses and was more pronounced in the last two doses. Despite the high adherence, advocacy is still needed to improve caretakers' understanding of the importance of adhering to the dose schedule in order to reduce the risk of building up resistant strains.

Authors' contributions: DOS, DK, GT, ZP, MP, MM and LLG designed the study protocol; DOS and DK supervised the data collection; DOS, MP and LLG analysed the data; all authors participated in drafting and revising the manuscript critically for intellectual content. All authors read and approved the final manuscript. DOS is guarantor of the paper.

Acknowledgements: The authors wish to thank the Muhimbili University of Health and Allied Sciences (MUHAS)/Karolinska Institutet collaboration for supporting the research of this study. They also wish to convey special thanks to Annika Janson for helpful advice and comments in the design of this study, to Mr Msangi for supervising the collection of blood samples, and to Mr Vicent Manyilizu for assisting in analysis of blood 
samples. Finally, they wish to convey their special gratitude to all participants, research assistants and others involved in support of the logistical arrangements for conducting this study.

Funding: The study on which this paper is based was funded by the Swedish International Development Corporation Agency/Department for Research Cooperation (SIDA/SAREC).

\section{Conflicts of interest: None declared.}

Ethical approval: Ethical approval was obtained from the Muhimbili University of Health and Allied Sciences (Dar es Salaam, Tanzania).

\section{References}

1. Hinton R, Auwun A, Pongua G, Davis M. Caregivers' acceptance of using artesunate suppositories for treating childhood malaria in Papua New Guinea. Am J Trop Med Hyg 2007;76:634-40.

2. Comoro C, Nsimba SE, Warsame M, Tomson G. Local understanding, perceptions and reported practices of mothers/guardians and health workers on childhood malaria in a Tanzanian district-implications for malaria control. Acta Trop 2003;87:305-13.

3. Makanga M, Premji Z, Falade C, Karbwang J, Mueller E, Andriano K, et al. Efficacy and safety of the six-dose regimen of artemetherlumefantrine in pediatrics with uncomplicated Plasmodium falciparum malaria: a pooled analysis of individual patient data. Am J Trop Med Hyg 2006;74:991-8.

4. Dondorp A, Yeung S, White L, Nguon C, Day N, Socheat D, et al. Artemisinin resistance: current status and scenarios for containment. Nat Rev Microbiol 2010;8:272-80.

5. Chinbuah A, Gyapong J, Pagnoni F, Wellington E, Gyapong M. Feasibility and acceptability of the use of artemether-lumefantrine in the home management of uncomplicated malaria in children 6-59 months old in Ghana. Trop Med Int Health 2006;11:1003-16.

6. Fogg C, Bajunirwe F, Piola P, Biraro S, Checchi F, Kiguli J, et al. Adherence to a six-dose regimen of artemether-lumefantrine for treatment of uncomplicated Plasmodium falciparum malaria in Uganda. Am J Trop Med Hyg 2004;71:525-30.

7. Beer N, Ali A, Rotllant G, Abass A, Omari R, Al-mafazy A, et al. Adherence to artesunate-amodiaquine combination therapy for uncomplicated malaria in children in Zanzibar, Tanzania. Trop Med Int Health 2009;14:766-74

8. Kabanywanyi A, Lengeler C, Kasim P, King'eng'ena S, Schlienger R, Mulure N, et al. Adherence to and acceptability of artemetherlumefantrine as first-line anti-malarial treatment: evidence from a rural community in Tanzania. Malar J 2010;9:48.

9. Simba D, Warsame M, Kakoko D, Mrango Z, Tomson G, Premji Z, et al. Who gets prompt access to artemisinin-based combination therapy? A prospective community-based study in children from rural Kilosa, Tanzania. PLoS One 2010;5:pii: e12104.

10. Krause G, Sauerborn R. Comprehensive community effectiveness of health care. A study of malaria treatment in children and adults in rural Burkina Faso. Ann Trop Paediatr 2000;20:273-82.

11. Nsungwa-Sabiiti J, Tomson G, Pariyo G, Ogwal-Okeng J, Peterson S. Community effectiveness of malaria treatment in Uganda-a long way to Abuja targets. Ann Trop Paediatr 2005;25:89-98.

12. Agyepong I, Ansah E, Gyapong M, Adjei S, Barnish G, Evans D. Strategies to improve adherence to recommended chloroquine treatment regimes: a quasi-experiment in the context of integrated primary health care delivery in Ghana. Soc Sci Med 2002;55:2215-26.

13. Ansah E, Gyapong J, Agyepong I, Evans D. Improving adherence to malaria treatment for children: the use of pre-packed chloroquine tablets vs. chloroquine syrup. Trop Med Int Health 2001;6: 496-504.
14. Gomes M, Faiz M, Gyapong J, Warsame M, Agbenyega T, Babiker A, et al. Pre-referral rectal artesunate to prevent death and disability in severe malaria: a placebo-controlled trial. Lancet 2009;373:557-66.

15. Nsungwa-Sabiiti J, Kallander K, Nsabagani X, Namusisi K, Pariyo G, Johansson A, et al. Local fever illness classifications: implications for home management of malaria strategies. Trop Med Int Health 2004;9:1191-9.

16. Simba D, Kakoko D, Warsame M, Premji Z, Gomes M, Tomson G, et al. Understanding caretakers' dilemma in deciding whether or not to adhere with referral advice after pre-referral treatment with rectal artesunate. Malar J 2010;9:123.

17. de Savigny D, Mayombana C, Mwageni E, Masanja H, Minhaj A, Mkilindi Y, et al. Care-seeking patterns for fatal malaria in Tanzania. Malar J 2004;3:27.

18. Eriksen J, Nsimba S, Minzi O, Sanga A, Petzoid M, Gustafsson L, et al. Adoption of the new antimalarial drug policy in Tanzania-a cross-sectional study in the community. Trop Med Int Health 2005; 10:1038-46.

19. Ministry of Health and Social Welfare (MoHSW). National guidelines for malaria diagnosis and treatment 2006. Dar es Salaam, Tanzania: MoHSW; 2006.

20. Ntale M, Ogwal-Okenga J, Mahindi M, Gustafsson L, Beck O. A fieldadapted sampling and HPLC quantification method for lumefantrine and its desbutyl metabolite in whole blood spotted on filter paper. J Chromatogr B Analyt Technol Biomed Life Sci 2008;876: 261-5.

21. National Bureau of Statistics (NBS Tanzania); Macro. Tanzania Demographic and Health Survey 2004-05. Calverton, MD: NBS and ORC Macro; 2005.

22. Yeung $\mathrm{S}$, White N. How do patients use antimalarial drugs? A review of the evidence. Trop Med Int Health 2005;10:121-38.

23. Marsh K. Malaria disaster in Africa. Lancet 1998;352:924-5.

24. Bell D, Wootton D, Mukaka M, Montgomery J. Measurement of adherence, drug concentrations and the effectiveness of artemetherlumefantrine, chlorproguanil-dapsone or sulphadoxine-pyrimethamine in the treatment of uncomplicated malaria in Malawi. Malar J 2009;8:204.

25. Price R, Uhlemann A, van Vugt M, Brockman A, Hutagalung R, Nair $S$, et al. Molecular and pharmacological determinants of the therapeutic response to artemether-lumefantrine in multidrug-resistant Plasmodium falciparum malaria. Clin Infect Dis 2006;42:1570-7.

26. Ezzet F, Van Vugt M, Nosten F, Looareesuwan S, White J. Pharmacokinetics and pharmacodynamics of lumefantrine (benflumetol) in acute falciparum malaria. Antimicrob Agents Chemother 2000;44:697-704.

27. Ashley E, Stepniewska K, Lindegardh N, Annerberg A, Kham A, Brockman A, et al. How much fat is necessary to optimize lumefantrine oral bioavailability? Trop Med Int Health 2007;12:195-200.

28. White N, van Vugt M, Ezzet F. Clinical pharmacokinetics and pharmacodynamics of artemether-lumefantrine. Clin Pharmacokinet 1999;37:105-25.

29. Obua C, Hellgren U, Ntale M, Gustafsson L, Ogwal-Okeng J, Gordi T, et al. Population pharmacokinetics of chloroquine and sulfadoxine and treatment response in children with malaria: suggestions for an improved dose regimen. Br J Clin Pharmacol 2008;4:493-501.

30. Piola P, Fogg C, Bajumirwe F, Biraro S, Grandessa F, Ruzagira E, et al. Supervised versus unsupervised intake of six-dose artemether-lumefantrine for treatment of acute, uncomplicated Plasmodium falciparum malaria in Mbarara, Uganda: a randomized trial. Lancet 2005;365:1467-73.

31. Simba D, Warsame W, Kimbute O, Kakoko D, Petzold M, Tomson G, et al. Factors influencing adherence to referral advice following prereferral treatment with artesunate suppositories in children in rural Tanzania. Trop Med Int Health 2009;14:775-83.

32. White N. Antimalarial drug resistance. J Clin Invest 2004;113: 1084-92.

33. Hildenwall H, Lindkvist J, Tumwine J, Bergqvist $Y$, Pariyo G, Tomson G, et al. Low validity of caretakers' reports on use of selected antimalarials and antibiotics in children with severe pneumonia at an urban hospital in Uganda. Trans R Soc Trop Med Hyg 2009;103: 95-101. 